\title{
LOW TEMPERATURE PLASMA APPLICATIONS IN MEDICINE
}

\author{
I K.-D. Weltmann1, H.-R. Metelmann², Th. von Woedtke ${ }^{1}$ - DOI: http://dx.doi.org/10.1051/epn/2016507 \\ $\square{ }^{1}$ Leibniz Institute for Plasma Science and Technology (INP Greifswald), Greifswald, Germany \\ ${ }^{2}$ Greifswald University Medicine, Greifswald, Germany
}

\begin{abstract}
The main field of plasma medicine is the direct application of cold atmospheric plasma (CAP) on or in the human body for therapeutic purposes. CAP is effective both to inactivate a broad spectrum of microorganisms including multiple drug resistant ones and to stimulate proliferation of mammalian cells. Clinical application has started in the field of wound healing and treatment of infective skin diseases.
\end{abstract}

\section{Cold atmospheric plasma (CAP) sources for medical application}

A well-established field of atmospheric plasma application is electro surgery where thermal plasma effects are used above all for coagulation and tissue cutting. In contrast, plasma medicine is focused actually on low temperature plasmas $\left(<40^{\circ} \mathrm{C}\right)$ to avoid thermal effects on living structures. Cold atmospheric plasma (CAP) sources for medical application have to meet particular requirements. Such devices have to guarantee manageable, stable, reliable, and reproducible operation at low temperature and open atmospheres. Besides a comprehensive characterization of qualitative and quantitative plasma parameters and "macroscopic" characteristics especially a meaningful knowledge of biological performance is an essential prerequisite for both effective and safe medical application.

During recent years, mainly three basic types of CAP devices were tested and partially applied for medical purposes (Fig. 1) [1,2,3].

In the volume dielectric barrier discharge (DBD) plasma is ignited in the gap between an isolated high voltage electrode and the target to be treated, i.e. in medical application human tissue (e.g. skin or wound surface) is part of the discharge electrode configuration. In the surface $\mathrm{DBD}$, plasma is ignited around an individually designed electrode structure (e.g. circular or grid-like) which is isolated from a counter electrode. Both electrodes can serve either as high voltage or ground electrodes. For treatment of living tissue, the plasma has to be brought in close vicinity of the target to be treated. With both DBD configurations, atmospheric air is usually the working gas.

In a plasma jet device, the electrode setup for plasma generation is located usually in a capillary or tube-like arrangement in most cases inside a pen-like device. Diverse electrode configurations can be used, e.g. pin electrodes, ring electrodes, plate electrodes etc. A working gas is flowing through the tube. The plasma is ignited inside the device. The effluent is blown out along the gas flow and can be brought into direct contact with the target to be treated. Several plasma jet devices are using noble gases like helium or argon, but air or gas mixtures are also useful as working gases.

Independent on the basic principle of plasma generation, all these atmospheric pressure plasmas are small scale and filamentary and are generated inside small discharge gaps ( $\mathrm{p}^{*} \mathrm{~d}$-scaling of breakdown voltage). The plasmas are non-uniform and constricted and consist of micro discharges or filaments, i.e. these are transient, short lived plasmas.

\section{Biologically active plasma components and basic mechanisms of action}

In general, cold atmospheric pressure plasma is a mixture of reactive components including charged species (ions and electrons), excited neutral species mainly from the working gas, reactive oxygen and nitrogen species, visible, ultraviolet (UV) and infrared (IR) radiation and other electromagnetic fields (Fig. 2). Dependent on the individual configuration of the plasma source, composition, relationship and quantity of these plasma compounds may vary significantly.

Based on a huge number of basic research using cultivated microorganisms and human cells [3], two main basic principles of biological plasma action have been identified recently:

1. Biological plasma effects are significantly caused by plasma induced changes of the liquid environment of cells.

2. Reactive oxygen and nitrogen species (ROS, RNS) generated in or transferred into liquid phases by plasma treatment play a dominating role in biological plasma activity. 
According to the actual knowledge, UV part of CAP has low or no direct biological effects because typically low doses are emitted by plasma devices designated for medical use. However, its supporting role in reactive species generation by photochemical activity has to be taken into consideration.

Electrical fields or current, respectively, reaching living tissue is strongly dependent on type of discharge and therefore might have varying direct biological effects. In this field, much more research is needed to finally enlighten the role of this plasma compound for its direct part in biological and medically relevant plasma action but also for its role in the generation or support of action of other plasma compounds, above all of reactive species [4].

However, the dominating role of ROS and RNS is established and demonstrated by several experimental setups independent on the specific plasma device used [5,6]. Generation of ROS and RNS is mainly referable to atmospheric oxygen and nitrogen which are part of the working gas in air-based plasma sources but is also admixed into the plasma in the case of noble gas-based plasma sources if they are working at open atmospheric conditions. According to the actual state of knowledge, differences of biological performance between plasma sources are mainly referred to quantities of ROS and RNS or its proportion of mixture in the respective plasma. However, possible role of UV radiation or electric fields has to be kept in mind.
The fundamental insight of the dominating role of ROS and RNS was highly valuable because the large and well established field of redox biology now can serve as a sound scientific basis to explain biological effects of CAP. ROS and RNS regularly occur in cell biological processes (e.g. superoxide $\mathrm{O}_{2} \bullet$, hydrogen peroxide $\mathrm{H}_{2} \mathrm{O}_{2}$, hydroxyl radical $\bullet \mathrm{OH}$, singlet oxygen ${ }^{1} \mathrm{O}_{2}$, nitric oxide $\bullet \mathrm{NO}$, nitrogen dioxide $\bullet \mathrm{NO}_{2}$ und peroxynitrite $\mathrm{ONOO}^{-}$). Therefore, mammalian cells have protective mechanisms to save from reactive species concentrations going beyond physiological levels. Such so-called oxidative stress might have severe consequences, e.g. genotoxic DNA changes. However, detailed investigations using well-established experimental procedures could demonstrate repeatedly that detrimental plasma effects on cells in general and particularly on DNA result either in cellular repair processes or in induction of programmed cell death (apoptosis) as a direct consequence. It has been demonstrated that application of cold atmospheric plasma does not cause increased risk for genotoxic effects $[7,8]$.

Three general biological plasma effects have been described repeatedly that are most relevant for medical application [3]:

- its potential to inactivate a broad spectrum of microorganisms including multidrug resistant ones

- its potential to stimulate cell proliferation and consequently to promote tissue regeneration
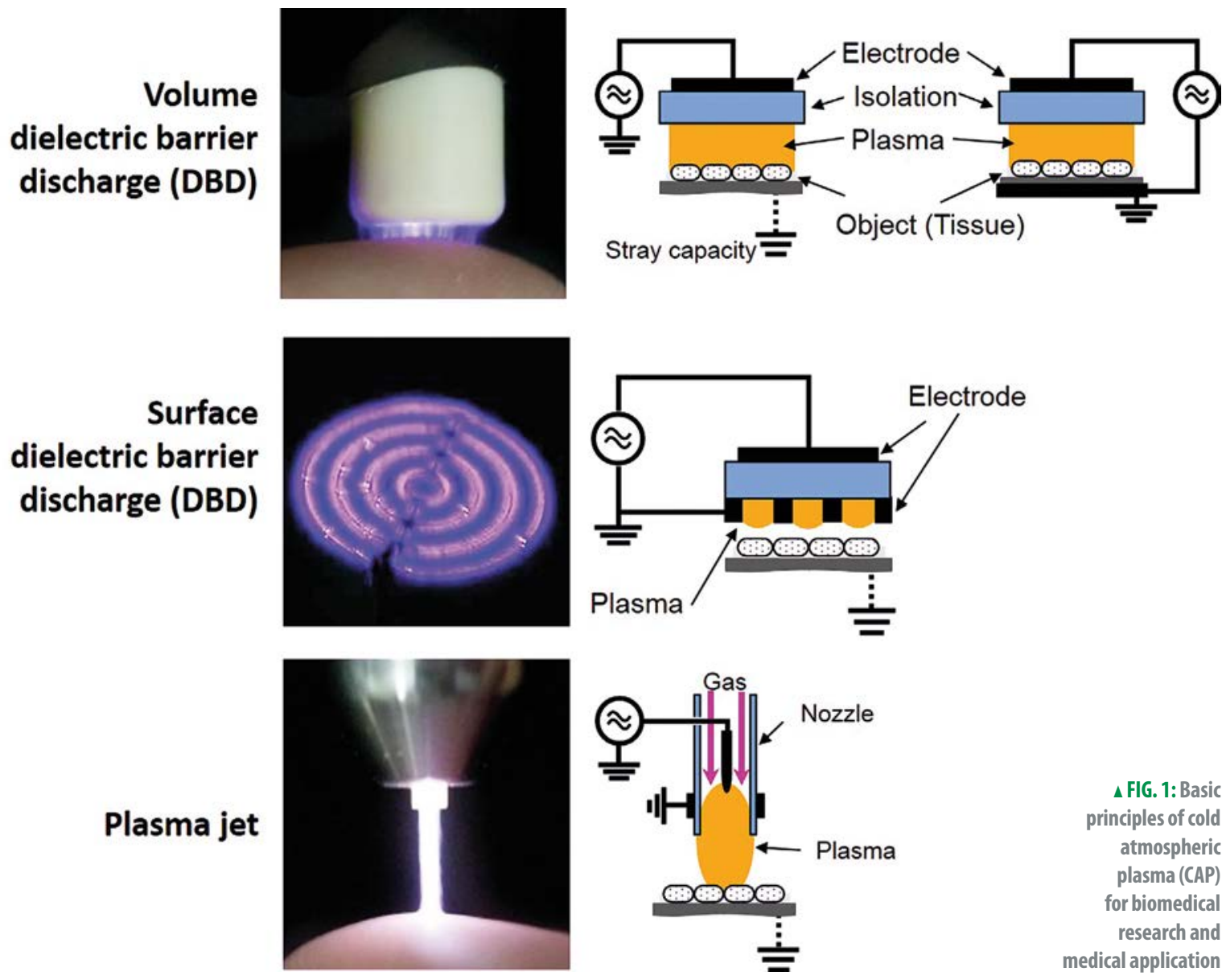


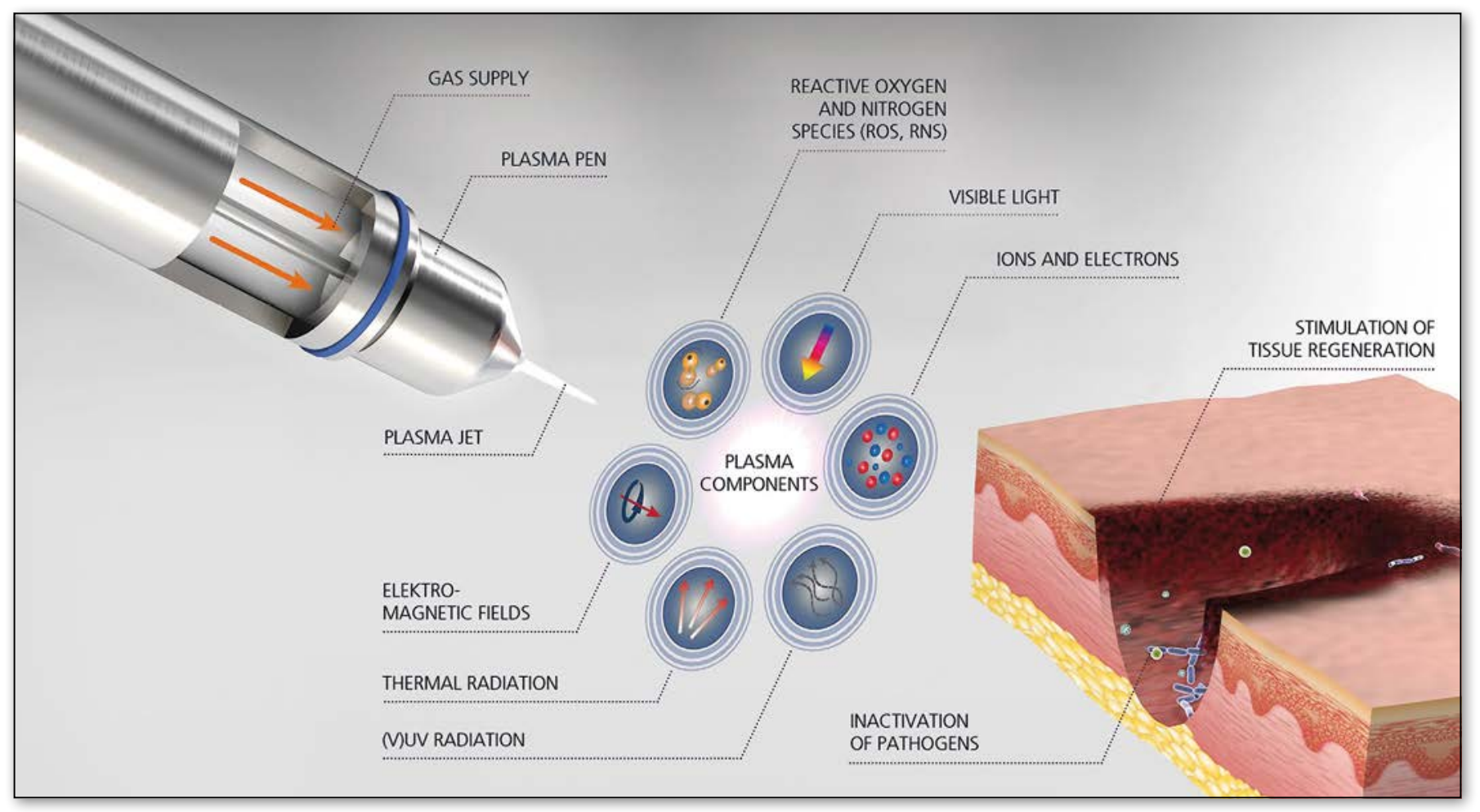

- its ability to inactivate mammalian cells and especially cancer cells by initialization of the programmed cell death (apoptosis)

\section{Medical application of CAP}

Since 2013, first CAP sources got CE certification as medical devices. One of it is the argon-driven cold atmospheric plasma jet kINPen MED (neoplas tools $\mathrm{GmbH}$, Greifswald, Germany), which is based on comprehensive physical, biological, pre-clinical and clinical characterization $[9,10]$. Two other well-investigated medical CAP devices are the jet-like microwave-driven Ar-plasma torch MicroPlasSter (ADTEC, Hunslow, UK) and the PlasmaDerm device (CINOGY GmbH Duderstadt, Germany) which is based on a volume DBD working with atmospheric air [11].

These plasma sources are certified mainly for the treatment of chronic wounds as well as pathogen-based skin diseases. The integrated concept of plasma-supported wound healing combines antimicrobial (antiseptic) plasma activity with a direct stimulation of tissue regeneration (Fig. 2). With these devices, routine application in medical practice in first clinics and doctor's offices above all in Germany has started. In the treatment of long-lasting chronic and infected wounds promising results are reported particularly in cases where conventional treatment fails. According to the feedback of the doctors that use it, a re-start or acceleration of wound healing process up to complete wound closure is reported in more than $80 \%$ of the patients as a preliminary result. Additionally, clinical users emphasize the CAP effectivity to eradicate multiple drug resistant bacteria (e.g. MRSA).
A highly topical field of basic and preclinical research is CAP application in cancer therapy due to the fact that CAP can inactivate cancer cells by induction of the programmed cell death (apoptosis). Because these cells seem to be much more sensitive for CAP treatment compared to non-malignant cells it opens up new options of supportive CAP application e.g. in surgical or radiative cancer eradication as well as in palliative cancer therapies $[12,13,14]$.

Possibilities of plasma application in dentistry include disinfection of tooth root canal, treatment of dental implants both for biofilm removal and improvement of bone cell adherence and therapy of intraoral infections and also wounds [15].

Besides these large fields of basic, pre-clinical and clinical research in plasma medicine, further fields of medical plasma use, such as ophthalmology, cardiology, pneumology or plastic and aesthetic surgery are investigated.

\section{Actual challenges and further prospects}

Despite the fact that first clinical application of CAP devices have already been realized, there are several needs to further improve and optimize this innovative plasma technology in medicine. There are both physical and technological but also biological and medical challenges.

Two main points have to be addressed in the next future: - adaptation of plasma devices for specific medical applications with regard to manageability under ergonomic and application site-related aspects;

- adaptation of plasma with regard to its composition to realize specific and selective biological effects. $\triangle$ FIG. 2: With cold atmospheric plasma (CAP) a mixture of reactive components dominated by reactive oxygen and nitrogen species (ROS, RNS) is working on living tissue, e.g. a wound. Wound healing by plasma is a combination of inactivation of bacteria and stimulation of tissue regeneration. 
For application-adapted plasma devices, several physical and technical concepts are existing for flat DBD-based plasma devices and plasma jet arrangements for large-area treatment, catheter-like plasma devices for endoscopic application as well as plasma devices to reach difficult to access areas in cavities e.g. for dental applications $[2,16,17]$.

For the adaptation of plasma composition to specific biological effects and subsequently to specific and selective medical applications, much more interdisciplinary research about detailed mechanisms of biological plasma effects and the specific role of the different plasma components, above all of the reactive species is needed. Furthermore, it is not known in detail how and in which extent the individual state of health of the patient influences the success of plasma therapy, which has to be taken into account to define individual treatment parameters.

Finally, perhaps the greatest challenge for the next future is to find or define a specific parameter or set of parameters for a device-independent control and monitoring of plasma treatment similar to the common "dose" in mainly irradiation-based physical therapies like laserand radiotherapy.

4

\section{The main field of plasma medicine is the direct application of cold atmospheric plasma (CAP) on or in the human body for therapeutic purposes. CAP is effective both to inactivate a broad spectrum of microorganisms including multiple drug resistant ones and to stimulate proliferation of mammalian cells. Clinical application has started in the field of wound healing and treatment of infective skin diseases. $\eta$}

Unique advantages of plasma application for therapeutic purposes are:

1. Active components are generated locally and only for the required duration of the application on-site primarily by a physical process.

2. Biologically active plasma components (above all reactive oxygen and nitrogen species) are the same as occur in regular physiological and biochemical processes in the body but cannot be supported adequately by drugs.

3. Because of its localized and short-term generation by local plasma treatment these substances can be detoxified by processes of regular cell metabolism, i.e. there is no increased risk of plasma application.

It can be expected that plasma medicine will become an independent and successful part of modern medicine within the next years. To attain this objective, more systematic clinical trials are essential to meet the demands of evidence based medicine.

\section{Acknowledgement}

The authors gratefully acknowledge the substantial financial support provided by the German Federal Ministry of Education and Research, the Ministry of Education, Science and Culture and the Ministry of Economics, Construction and Tourism of the State of Mecklenburg-Western Pomerania (Germany) as well as the European Union, European Social Fund.

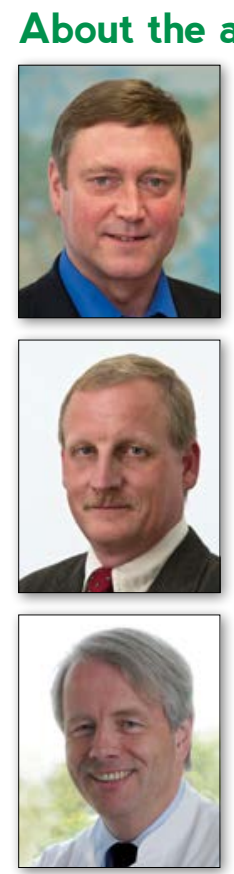

uthors

Klaus-Dieter Weltmann and Thomas von Woedtke are from Leibniz Institute for Plasma Science and Technology (INP), Greifswald, Germany. Klaus-Dieter Weltmann is Chairman of the Bord of INP and Head of the Research Division Plasmas for Environment and Health. He held a Professorship for Experimental Physics at Greifswald University and a Visiting Professorship at New York University. Thomas von Woedtke is Program Manager Plasma Medicine at INP and Professor for Plasma Medicine at Greifswald University Medicine. Hans-Robert Metelmann (M.D., D.M.D., Ph.D.) is Professor and Head of Department of Oral and Maxillofacial Surgery/Plastic Surgery at Greifswald University Medicine, Greifswald, Germany.

\section{References}

[1] K.-D. Weltmann et al., Pure Appl. Chem. 82, 1223 (2010)

[2] G.Y. Park et al., Plasma Sources Sci Technol 21, 043001 (2012)

[3] Th. von Woedtke et al., Phys. Rep. 530, 291 (2013)

[4] T. Darny et al., 2015 IEEE International Conference on Plasma Sciences (ICOPS); DOI: 10.1109/PLASMA.2015.7179640

[5] D.B. Graves, J. Phys. D: Appl. Phys. 45, 263001 (2012)

[6] D.B. Graves, Clin. Plasma Med. 2, 38 (2014)

[7] K. Wende et al., Cell Biol. Int. 38, 412 (2014)

[8] K. Wende et al., Mutat. Res. Genet. Toxicol. Environ. Mutagen. 798, 48 (2016)

[9] K.-D. Weltmann et al., Contrib. Plasma Phys. 49, 631 (2009)

[10] S. Bekeschus et al., Clin. Plasma Med. 4, 19 (2016)

[11] G. Isbary et al., Expert Rev. Med. Devices 10, 367 (2013)

[12] A.M. Hirst et al., Tumor Biol. 27, 7021 (2016)

[13] M. Keidar, Plasma Sources Sci. Technol. 24, 033001 (2015)

[14] M. Schuster et al., J. Cranio Maxill. Surg. (2016); DOI: 10.1016/j. jcms.2016.07.001

[15] S. Cha and Y.-S. Park, Clin. Plasma Med. 2, 4 (2014)

[16] K.-D. Weltmann et al., Contrib. Plasma Phys. 54, 104 (2012)

[17] K.-D. Weltmann et al., IEEE Trans. Plasma Sci. 40, 2963 (2012) 